\title{
Aptamer Grafting onto (on) and into (in) Pegylated Gold Nanoparticles: Physicochemical Characterization and In vitro Cytotoxicity Investigation in Renal Cells
}

Celia Arib 1 , Serena Milano², Andrea Gerbino² and Jolanda Spadavecchia ${ }^{\text {** }}$

${ }^{1}$ CNRS, UMR 7244, CSPBAT, Laboratoire de Chimie, Structures et Propriétés de Biomateriaux et d'Agents Therapeutiques, Université Paris 13, Sorbonne Paris Cité, Bobigny, France

${ }^{2}$ Department of Biosciences, Biotechnologies and Biopharmaceutics, University of Bari, 70126 Bari, Italy

\begin{abstract}
Gold Nanoparticles (AuNPs) have already a remarkable interest as viable biomedical materials. Additionally, the strategy of using biomolecules to modify their surface properties is a very attractive as it leads to the generation of new nanometric hybrid materials. In this respect, aptamers, functional small single-strand oligonucleotides (DNA or RNA), are ideal candidates for molecular targeting applications since the high affinity to their target molecules. Thus, the urge of new and effective methodologies to graft aptamers on AuNPs is rapidly increasing especially for applications in bioanalysis and biomedicine, including early diagnosis and drug delivery. Here we used two chemical methodologies to conjugate the aptamer (APT) onto pegylated gold nanoparticles (PEG-AuNPs): the carbodiimide chemistry (EDC/NHS) (methodology ON) and the chelation-bond (R-Au bond) (methodology IN). The aptamer's conjugations with the PEGAuNPs were characterized by UV-Vis absorption, Raman Spectroscopy and transmission electron microscopy (TEM). In addition, the potential nanotoxicity of the two aptamer-conjugated AuNPs was evaluated on two different renal cell lines, being the kidneys one of the most important site of bioaccumulation upon systemic circulation. Interestingly, the two aptamer-conjugated AuNPs showed different cytotoxicity when exposed to human embryonic kidney (HEK293) and mouse collecting duct cells (M-1), indicating that cell viability has to be taken into account when choosing the proper strategy for NPs production. In conclusion this study provides two effective methods to graft aptamers on NPs and important insights regarding NPs conformation and the relative cell viability.
\end{abstract}

Keywords: Colloids; Functionalization; Biological interaction; Biomarker; PEG-AuNPs; Raman Spectroscopy; Aptamer; SERS

\section{Introduction}

During the last years, the nanoparticles were intensively used in industrial and medical applications [1-3]. Among them, Gold Nanoparticles (AuNPs) have a remarkable interest as viable biomedical materials and the research efforts about this subject are continuously growing due to their unique physical and chemical properties [4]. By using some of the biomolecules to modify the surface properties is a very attractive strategy, as it leads to a new generation of nanometric hybrid materials [5-7]. Several characteristics of such nanostructures have been identified as providing a better biocompatibility and furtivity to the nanoparticles or a better targeting of some specific disease biomarkers for nanosensor development or of some bio-structures as tumours or organs that have to be detected or treated [8]. Additionally, the physicochemical characteristics of NPs deeply impact their ability to migrate away from the organism's entry site [9]. Liver, spleen and kidney possess a fenestrated endothelium that allows for passive accumulation of NPs and are potential sites for NPs off-target toxicity or target of the therapy [10]. In particular, biodistribution studies in vivo confirmed renal distribution of NPs [11-14] and this highlight the need of evaluating nanotoxicity at renal level. One of the most promising bioreceptors is the DNA aptamer, a DNA single strand (ssDNA) specially designed to have a high affinity to proteins [15] or other kinds of analytes as toxins $[16,17]$. Based on their high binding affinity and specificity towards other molecules, aptamers, can be utilized in applications ranging from biosensing to diagnostics and therapeutics [18-20]. Meanwhile, advances in nanotechnology have led to new and improved materials for biomedical applications [21]. Specifically, nanoparticles can readily interact with both intra- and extra-cellular biomolecules to yield improved signal amplification and target recognition [22]. To accomplish this, several parameters were investigated, including nanoparticle size, conjugation chemistry, use of multiple aptamer sequences on the nanoparticles, and use of multiple nanoparticles with different aptamer sequences $[16,18]$. In this context the study of the interaction between the bioreceptor (e.g. aptamer) and proteins is essential to improve the biomedical application of nanoparticles [23,24]. Among various analytes of interest, aquaporins (AQPs) are membrane water/glycerol channels that plays a key role in many physiological functions [25], as well as chemical tools to assess AQPs function in biological systems $[25,26]$. In recent years several gold (III) compounds have shown promising anticancer effects related to the inhibition of different protein targets, such as the proteasome and specific zinc finger proteins $[27,28]$.

In particular, Soveral $G$ et al. have described the selective and potent inhibitory effect (in the nanomolar range) of $\mathrm{Au}$ (III) complexes bearing nitrogen donor ligands on AQPs proteins, which together with their limited toxicity and high water solubility makes them suitable candidates for future in vivo studies [27]. The aim of the current paper, is to preliminary inquire the possibility to exploit the bioconjugation

*Corresponding author: Jolanda Spadavecchia, CNRS, UMR 7244, CSPBAT, Laboratoire de Chimie, Structures et Propriétés de Biomateriaux et d'Agents Therapeutiques, Université Paris 13, Sorbonne Paris Cité, Bobigny, France, Tel: 33148388526; E-mail: jolanda.spadavecchia@univ-paris13.fr

Received: October 24, 2018; Accepted: November 14, 2018; Published: November 19, 2018

Citation: Arib C, Milano S, Gerbino A, Spadavecchia J (2018) Aptamer Grafting onto (on) and into (in) Pegylated Gold Nanoparticles: Physicochemical Characterization and In vitro Cytotoxicity Investigation in Renal Cells. J Nanomed Nanotechnol 9: 520. doi: 10.4172/2157-7439.1000520

Copyright: (C) 2018 Arib C, et al. This is an open-access article distributed under the terms of the Creative Commons Attribution License, which permits unrestricted use, distribution, and reproduction in any medium, provided the original author and source are credited. 
of aptamer AQP-2 onto gold nanoparticle surface as two different chemical strategies in order to evaluate their biological interactions with kidney cells as well as the chemical physical characterization of AQP2 aptamer onto gold nanoparticles. In particular, two methods for the aptamer grafting onto pegylated gold nanoparticles (PEG-AuNPs) was established: the carbodiimide chemistry [29] (reaction between $\mathrm{COOH}$ and $\mathrm{NH}_{2}$ chemical groups) and, for the first time, the chelation reaction concerning the complexation between $\mathrm{Au}-\mathrm{Cl}$ and aptamer functional groups $\left(\mathrm{PO}_{4}^{3-} ; \mathrm{CO}\right.$ and/or $\left.\mathrm{NH}_{2}\right)$.

Both aptamer-conjugated PEG-AuNPs underwent physicochemical characterization and tested for cytotoxicity on two different renal cell lines, human embryonic kidney (HEK293) and mouse collecting duct cells (M-1), respectively.

This study open the way to develop original protocols will be employed for decoration of hybrid nanoparticles in order to create aptamer-based nanomaterials, with high affinity cancer biomarkers.

\section{Materials and Methods}

Tetrachloroauric acid $\left(\mathrm{HAuCl}_{4}\right)$, sodium borohydride $\left(\mathrm{NaBH}_{4}\right)$, dicarboxylic PolyEthylene Glycol (PEG)-600 (PEG), N-hydroxysuccinimide (NHS), 1-(3-dimethylaminopropyl)-N'ethylcarbodiimide hydrochloride (EDC) and phosphate-buffered solution (PBS) were all provided by Sigma Aldrich at maximum purity grade. Aquaporin 2 aptamer was purchased from Eurogentec.

\section{Aquaporin 2 aptamer(APT)}

Aquaporin 2 Homo sapiens aptamer was purchased from Eurogentec. The aptamer was dispersed in PBS buffer $(\mathrm{pH}=9)$.

\section{Cell culture and in vitro cytotoxicity assay}

Human embryonic kidney cells HEK293 cells were cultured in DMEM Glutamax supplemented with $10 \%$ fetal bovine serum and 100 i.u. $/ \mathrm{ml}$ penicillin, $100 \mu \mathrm{g} / \mathrm{ml}$ streptomycin at $5 \% \mathrm{CO}_{2}$ and $37^{\circ} \mathrm{C}$. Mouse cortical collecting duct M-1 cells [30] were maintained in DMEM/F12 (1:1) supplemented with $5 \%$ fetal bovine serum, $2 \mathrm{mM} \mathrm{L}$-glutamine, 100 i.u./ml penicillin, $100 \mu \mathrm{g} / \mathrm{ml}$ streptomycin, and $5 \mu \mathrm{M}$ dexamethasone. Cells were either left under basal condition or exposed to PEG-AuNPs $(1 \mu \mathrm{M})$, APT ON PEG-AuNPs $(1 \mu \mathrm{M})$ and APT IN PEG-AuNPs $(1 \mu \mathrm{M})$ for $6,12,24,48$ and 72 hours at $37^{\circ} \mathrm{C}$ to evaluate cytotoxicity using trypan blue exclusion assay. Briefly, an aliquot of the cell suspension was diluted $1: 1(\mathrm{v} / \mathrm{v})$ with $0.4 \%$ trypan blue and viable cells were counted with a hemocytometer Luna II automated cell counter (Logos Biosystem). The percentage viability was calculated by dividing the number of viable cells by the number of all counted cells. Three independent experiments were carried out. Cell viability data were analyzed using Prism software (version 6.0; www.graphpad.com). Statistical differences between experimental conditions were determined by One-way Anova with Tukey's multiple comparisons post-test. Data were expressed as means \pm SEM and $p<0.05$ was considered statistically significant.

\section{Synthesis of PEG-AuNPs}

Colloids of COOH-terminated PEG-coated AuNPs (PEG-AuNPs) were prepared by a well assessed chemical reduction process according to previously described procedure (Scheme 1) [2].

\section{Determination of PEG-AuNPs concentration}

AuNPs concentration was determined by exploiting standard mathematical calculations in colloidal solution as described previously [31].

\section{Bioconjugation of PEG-AuNPs with aptamers}

PEG-AuNPs surface was modified by APT aptamer, according to the grafting procedures depicted in Scheme 1.

\section{Aptamer graftings onto peg-aunps by carbodiimide cross- linker chemistry (apt on peg-aunps)}

The first grafting strategy consists of the conjugation of the AQP2 aptamer (APT) or on the PEG-AuNPs surface by carbodiimide a)
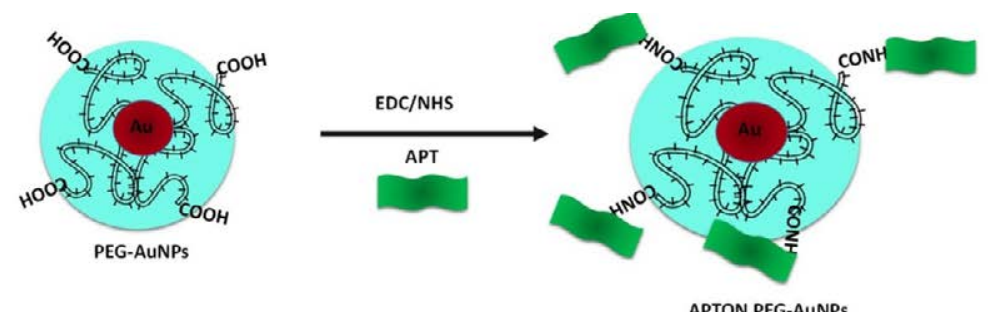

APTON PEG-AUNPS
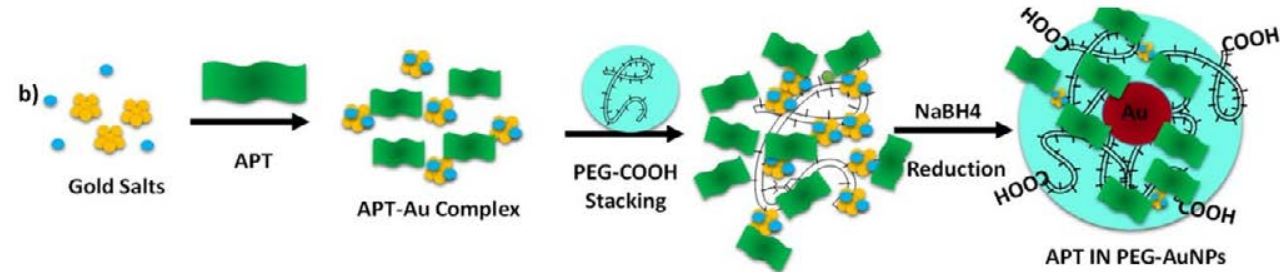

APT IN PEG-AUNPS

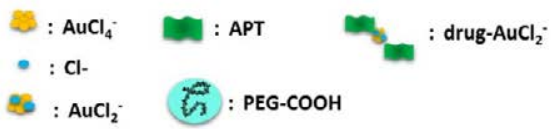

Scheme 1: Schematic representation of the synthesis of a)APT ON PEG-AuNPs and b) APT IN PEG-AuNPs via carbodiimide chemistry (a) and chelation reaction (b) (Please note that drawings are not in scale and are not intended to be representative of the full samples composition). 
Citation: Arib C, Milano S, Gerbino A, Spadavecchia J (2018) Aptamer Grafting onto (on) and into (in) Pegylated Gold Nanoparticles: Physicochemical Characterization and In vitro Cytotoxicity Investigation in Renal Cells. J Nanomed Nanotechnol 9: 520. doi: 10.4172/2157-7439.1000520

Page 3 of 10

chemistry. Briefly, $50 \mu \mathrm{l}$ of EDC/NHS (40/10 w/w ratio) aqueous solution was added into $2 \mathrm{ml}$ PEG-AuNPs dispersion (42 mM). After 2 h, $50 \mu$ of aptamer solution $(10 \mu \mathrm{M}$ in PBS $\mathrm{pH}$ 9) was added in $2 \mathrm{ml}$ of the reaction mixture and stirred for $2 \mathrm{~h}$ at room temperature. The APTPEG-AuNPs (6APT/NPs) thus obtained were centrifuged two times at $9,000 \mathrm{rpm}$ for $10 \mathrm{~min}$ to remove excess of no-conjugated APT and dried under nitrogen.

\section{Aptamer graftings into peg-aunps by complexation} methodology (apt in peg-aunps)

The second grafting strategy consists of the complexation of the AQP2 APT aptamer (50 $\mu$ l of APT $10 \mu \mathrm{M}$; PBS pH 9)(APT) with gold salt $\left(\mathrm{HAuCl}_{4}\right)\left(5 \mathrm{ml} 2.6^{\star} 10^{-4} \mathrm{M}\right)$, and $50 \mu \mathrm{l}$ of PEG-diacide (PEG)( 1 $\mathrm{mM}$ ) thought chelation reaction, in order to form APT IN PEG-AuNPs. After $18 \mathrm{~h}$ the APT IN PEG-AuNPs suspension was centrifuged twice at $6000 \mathrm{rpm}$ for $10 \mathrm{~min}$ to remove excess of aptamer and then the pellets were redispersed in $1 \mathrm{~mL}$ MilliQ water. The resultant colloidal solution was sonicated for 5 minutes and then stirred for $1 \mathrm{~h}$ at room temperature.

\section{Physico-chemical characterization}

All the measurements were performed in triplicate in order to validate the reproducibility of the synthetic and analytical procedures.

UV/Vis measurements: Absorption spectra were recorded using a Perkin Elmer Lambda UV/Vis 950 spectrophotometer in plastic cuvettes with an optical path of $10 \mathrm{~mm}$. The wavelength range was $200-900 \mathrm{~nm}$.

Transmission electron microscopy (TEM): TEM images were acquired with a JEOL JEM 1011 microscope (JEOL, USA) at an accelerating voltage of $100 \mathrm{kV} .2 \mu \mathrm{l}$ of the particle suspension was placed on a carbon coated copper grid (Smethurst High-Light Ltd) and dried at room temperature.

Raman spectroscopy: The Raman experiments have been performed on an Xplora spectrometer (Horiba Scientifics-France). The Raman spectras have been recorded using an excitation wavelength of $785 \mathrm{~nm}$ (diode laser) at room temperature. For measurements in solution, a macro-objective with a focal length of $40 \mathrm{~mm}(\mathrm{NA}=0.18)$ was used in backscattering configuration. The achieved spectral resolution is close to $2 \mathrm{~cm}^{-1}$.

Dynamic light scattering (DLS) and Zeta potential measurements: The size and zeta potential measurements were performed using a Zetasizer Nano ZS (Malvern Instruments, Malvern, UK) equipped with a He-Ne laser $\left(633 \mathrm{~nm}\right.$, fixed scattering angle of $\left.173^{\circ}\right)$ at room temperature.

\section{Stability of APT AuNPs (APT ON PEG-AuNPs; APT IN PEG- AuNPs) as a function of $\mathrm{pH}$}

For stability studies, APT AuNPs were dispersed in PBS (0.1 M; pH 7 and 5.5), and absorption spectra collected over 3 months (Figure S1).

Aptamer loading efficiency: The amount of the AQP2 aptamer (APT) incorporated onto APT ON PEG-AuNPs andAPT IN PEGAuNPs, were measured by UV-Vis absorption spectroscopy. Absorption at $260 \mathrm{~nm}$ was used to extrapolate APT concentrations based on a calibration curve (Figure S2). The aptamer loading efficiency was calculated as follows Equation 1:

$$
\text { APTloading efficiency }(\%)=\frac{C_{1}-C_{2}}{C_{1}} \times 100
$$

Where $C_{1}$ is the initial drug content and $C_{2}$ is the amount of free
A)
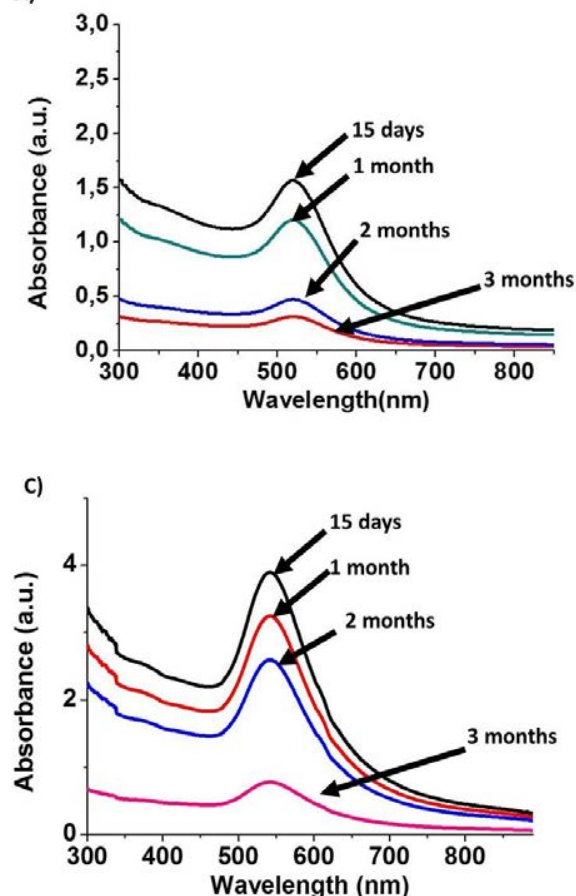
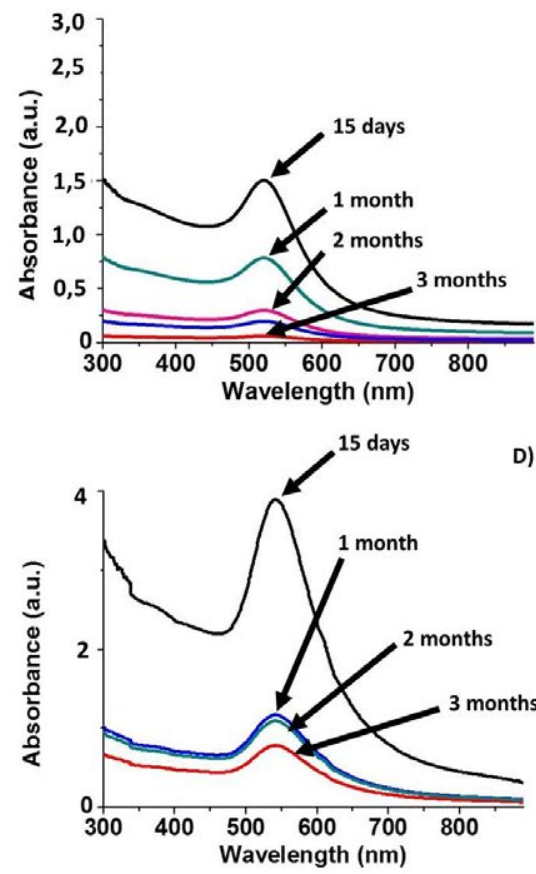

Figure S1: Changes in the UV-Vis absorption spectra of APT AuNPs when incubated in buffer solution at pH 7 (A: APT ON PEG-AuNPs; C: APT IN PEG-AuNPs) and pH 5.5 (B: APT ON PEG-AuNPs; D: APT IN PEG-AuNPs) up to three months. 
A)

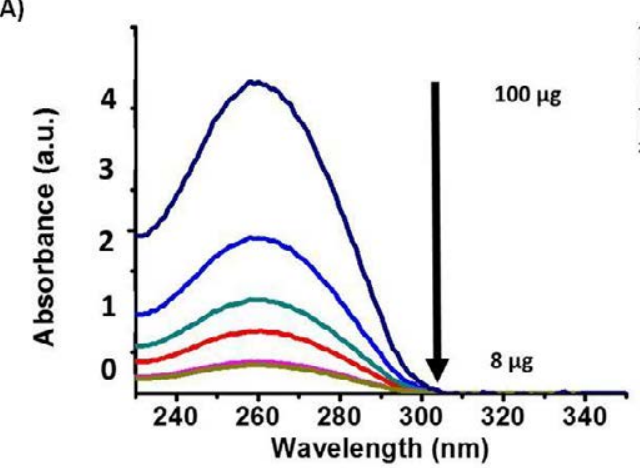

B)

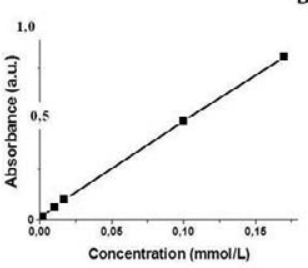

Figure S2: (A) UV-Vis absorption spectra of APT at increasing known concentrations. (B) A calibration curve $(R 2=0.9997)$ was extrapolated from such measurements. The amount of APT molecules contained into APT ON PEG-AuNPs, APT ON PEG-AuNPs were estimated based on this curve.

APT in the filtrate after separation of the nanoparticles by ultrafiltration with an Ultrafree MC centrifugal filter units, (30,000 NMWL, Millipore, USA).

Aptamer release from AuNPs: APT releases was evaluated at physiological temperature $\left(37^{\circ} \mathrm{C}\right.$ ). aptamer-loaded AuNPs ( APT ON PEG-AuNPs; APT IN PEG-AuNPs) were dispersed at concentration of $1.5 \times 10^{12}$ particles $/ \mathrm{ml}$ in $1.5 \mathrm{ml}$ PBS and performed by dialysis as previously described [32]. The concentration of aptamer released from AuNPs was expressed as a percentage of the total aptamer concentration present in the sample Equation 2 and plotted as a function of time.

$$
\% \text { APT released }=\frac{\text { Concentrationofaptamerreleased }}{\text { Initial aptamerconcentration }} \times 100
$$

\section{Endotoxin content in drug-gold nanoparticles}

As routine quality control of the materials prior to biological testing, the Pierce LAL Cromogenic Endotoxin Quantitation kit (Thermoscientific, France) was used to determine the LPS contamination in pegylated gold nanoparticles ( PEG-AuNPs) before and after conjugation with AQP2 aptamer(APT ON PEG-AuNPs; APT IN PEGAuNPs ) as described previously [33]. Results are reported in Table 1 as average $\left(\mathrm{n}_{\text {test }}=2\right) \pm$ standard deviation. Endotoxin contamination results were kept into account when defining the type of cytotoxic endpoint to be examined.

\section{Results and Discussion}

\section{Formation mechanism of APT AuNPs ( APT ON PEG- AuNPs; APT IN PEG-AuNPs)}

Previously, Spadavecchia et al. have synthetized and conjugated pegylated gold nanoparticles (PEG-AuNPs) with biomolecules (protein, antibody, peptides, aptamer) by several grafting methodologies $[2,5,7,31]$. Chemical-physical evaluations and biological "in vitro" studies of biomolecules onto pegylated-gold-nanoparticles have successfully showed a large influence in its therapeutic activity [32-35].

Recently, new nano-therapeutic agents based on drugs-goldcomplex such as doxorubicin, docetaxel, paclitaxel, gemcitabine has been generated [32,36,37]. According to this experimental approach called "Method IN", the drug interacts actively with gold salt $\left(\mathrm{HAuCl}_{4}\right)$ by chelation bonding and a biocompatible polymer molecules (PEG diacid) as surfactant, in order to form hybrid gold nanoparticles under reduction with $\mathrm{NaBH}_{4}$.
Other authors conjugated many type of aptamers in order to study the interaction with specific biomarkers [38]. Herein we designed and characterized the grafting of the AQP2 aptamer (APT) at the surface of the PEG-AuNPs for carbodiimide chemistry (method ON) and chelation bond (method IN). In the first case, APT was grafted on the surface of pegylated nanoparticles through the formation of amide links between the $\mathrm{COOH}$ groups embedding the surface of the PEGAuNPs and the $\mathrm{NH}_{2}$ groups of the Aptamer (Scheme 1 panel a previous activation of EDC/NHS). In the second case, APT participate to the stabilization of AuNPs via complexation and electrostatic interaction between its chetone and amino groups with chloride auric ions and dicarboxylic pegylated chains.

In particular, the formation of gold NPs from $\mathrm{AuCl}_{4}$ - includes some mains steps (Scheme $1 b$ ):

(1) Complexation of $\mathrm{APT}-\mathrm{AuCl}_{4}^{-}$and generation of gold clusters [5].

(2) Initial reduction of APT metal complex to form Au II;

(3) Staking process of polymer molecules by electrostatic adsorption onto the PEGylayed gold clusters;

(4) Final reduction of metal ions to form gold particles stabilized by molecules of APT.

In the first step, a mixture of APT molecules was added in $\mathrm{HAuCl}_{4}$ aqueous solution to induce formation of complexes. Then, the positively charged APT in water solution shows strong electrostatic interaction with the negatively charged $\mathrm{AuCl}_{4}^{-}$ions leading to formation of a hybrid complex APT-AuCl ${ }_{4}^{-}$(scheme 1b). The addition of PEG-COOH on the hybrid complex stabilizes it through electrostatic interaction between carbonyl and amino groups, improving the kinetics of reduction by complexation of Au ions [39] and controlling the growth process of the final nanoparticles (APT IN PEG-AuNPs). The steric arrangement of APT INPEG-Au clusters before reduction of $\mathrm{HAuCl}_{4}$ was confirmed by UV-Vis absorption spectroscopy, TEM and Raman Spectroscopy, as reported in the following sections.

\section{Comparative Aptamer grafting: physicochemical evaluation}

The UV-visible spectra of PEG-AuNPs exhibited a surface plasmon band at $515 \mathrm{~nm}$ (Figure 1, black line). After the APT binding onto the PEG-AuNPs (APT ON PEG-AuNPs) via EDC/NHS, the plasmon band is red-shifted to $523 \mathrm{~nm}$ (Figure 1, red line). Besides, we assume that 
Citation: Arib C, Milano S, Gerbino A, Spadavecchia J (2018) Aptamer Grafting onto (on) and into (in) Pegylated Gold Nanoparticles: Physicochemical Characterization and In vitro Cytotoxicity Investigation in Renal Cells. J Nanomed Nanotechnol 9: 520. doi: 10.4172/2157-7439.1000520

Page 5 of 10

the red-shift amplitude depends on both the type of bioconjugation method and the APT model. Concerning the chelation method, the redshift is bigger (to $543 \mathrm{~nm}$ ) with a decrease in intensity and a significant broadening (Figure 1, green line). The red-shift can be displayed by modification of dielectric environment of the nanoparticles thus confirming the successful functionalization of the AuNPs surface in both cases. In particular, the prominent red shift and the broadening observed for APT IN PEG-AuNPs can be due to agglomeration between the pegylated gold nanoparticles combined to the formation of classical van der Waals inter-aptamer interactions [40], thus the different steric conformation of APT during the chelation method. The different redshift of the plasmon band suggests that each chemical methodology (ON and IN) induce a specific chemical behavior of the APT from PEG-AuNPs. This will have considerable repercussions on the steric conformation of the APT at the PEG-AuNP surface as well as different immobilization kinetic [41,42]. Indeed, the chemical conjugation will influence the grafting position of the APT. With the carbodiimide (EDC/NHS) grafting, the APT is located directly at the gold surface decorated by a PEG layer whereas for the chelation method, the APT will be located into the core of gold, surface embedded of the PEG layer. This chemical behavior is due to a different adsorption onto gold facets of APT, based on their different steric conformation of chemical groups during nucleation and growth process of PEG-AuNPs. Consequently, the color bright violet-blue of the hybrid nanoparticles and the UV-Vis spectra remain unaltered after storage for more than three months at room temperature suggesting the formation of stable colloids suspension. Such observation is very remarkable since the nature of capping layer onto AuNPs plays a key role on the success of bioconjugation and comparative kinetic [5]. This behavior was confirmed by TEM Images. Figure 1-B panel 1 shows a spherical pegylated gold nanoparticles utilized as control with a diameter about $10 \pm 2 \mathrm{~nm}$ as described previously [2,5]. After carbodiimide grafting, the resulting APT ON PEG-AuNPs appears as circular agglomerate of nanoparticles embedded of a dense layer of polymer (Figure 1-B panel 2). Considering that the size of single aptamer was about $5 \mathrm{~nm}$ [43], we suppose that APT assume a preferential location of PEG molecules onto the particles, with a specific steric arrangement. Contrarily to shape of APT ON PEG-AuNPs,, APT IN PEG-AuNPs displays a snowlike shape, embedded in a shell of PEG, with a similar diameter around $50 \pm 2 \mathrm{~nm}$ (Figure 1-B panel 3. Spadavecchia et al. have realized the synthesis of analogous nanostructures using dicarboxylic PEG $[32,33]$ and drugs, while characteristic snowflakes nanoparticles were obtained
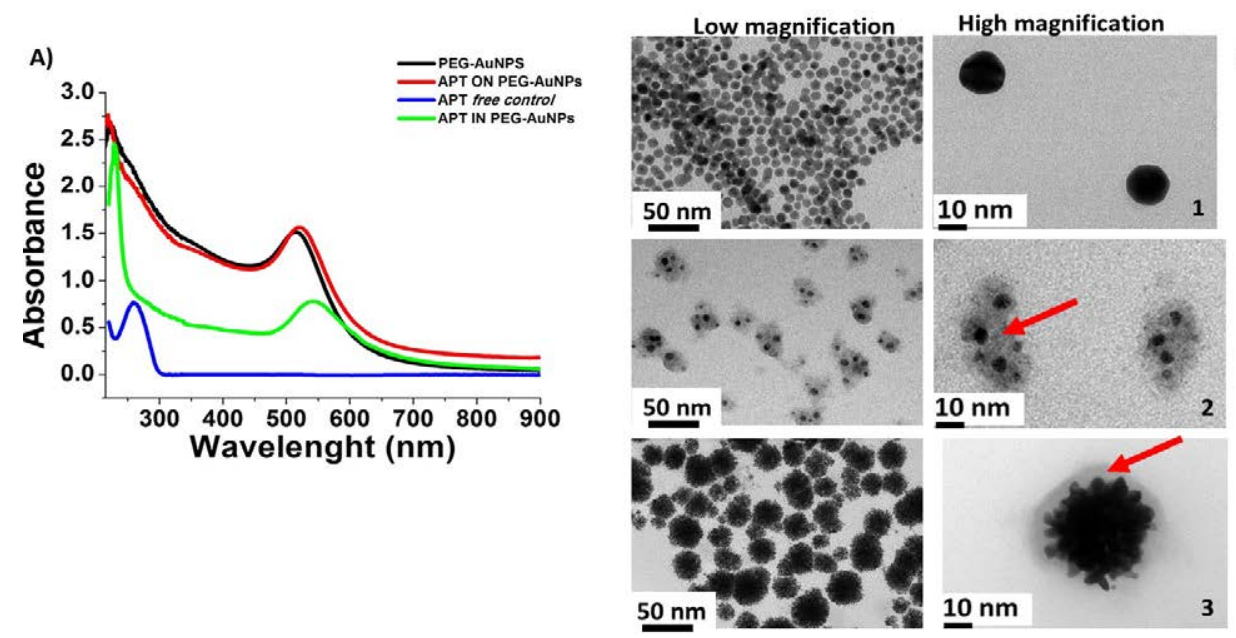

B)

Figure 1: (A) Normalized UV-Vis absorption spectrum of APT ON PEG-AuNPs (red line), APT IN PEG-AuNPs( green line) and PEG-AuNPs ( black line) APT ( blue line) as control, (B) TEM images of PEG-AuNPs before (panel 1) and after conjugation of APT by carboddiimide chemistry (panel 2) and chelation reaction (panel 3). Scale bars: $50 \mathrm{~nm}$; $10 \mathrm{~nm}$ (low and high magnification). Directional arrows showing the presence of an organic APT-PEG layer of few nanometres.
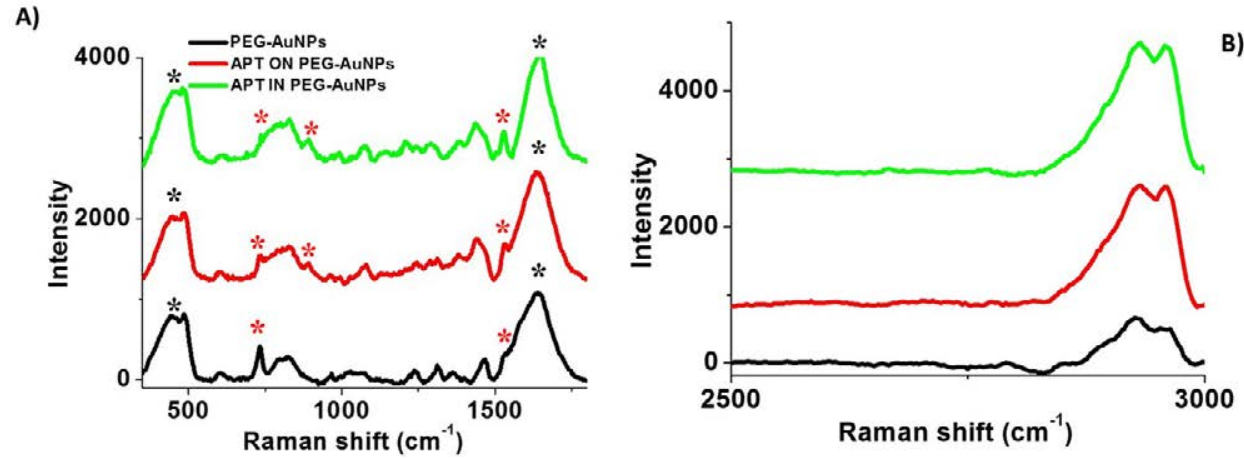

Figure 2: (A-B) Raman spectra of the APT ON PEG-AuNPs (green line) and APT IN PEG-AuNPs (red line) compared to free PEG-AuNPs (black line). Experimental conditions: $\lambda_{\mathrm{exc}}=785 \mathrm{~nm}$; laser power $20 \mathrm{~mW}$; accumulation time $180 \mathrm{~s}$. 
Citation: Arib C, Milano S, Gerbino A, Spadavecchia J (2018) Aptamer Grafting onto (on) and into (in) Pegylated Gold Nanoparticles: Physicochemical Characterization and In vitro Cytotoxicity Investigation in Renal Cells. J Nanomed Nanotechnol 9: 520. doi: 10.4172/2157-7439.1000520

by incorporating protoporphyrin molecules in the growth solution of AuNPs [44].

According to previous studies [5,32,45], when APT was mixed to gold salt $\left(\mathrm{AuCl}_{4}^{-}\right)$complex, the PEG adapt its chemical conformation switching from mushroom to brush mode conformation.

The APT binding is confirmed by DLS and Zeta potential measurements (Table 1). The hydrodynamic diameter is increased by around $10 \mathrm{~nm}$ which means an increase of $5 \mathrm{~nm}$ of the radius. This distance should correspond to a superior value of the aptamer length since the hydrodynamic radius includes the interaction with water and could not reflect the exact diameter. Moreover, Zeta potential measurements show that PEG-AuNPs and APT PEG-AuNPs were colloidally stable at physiological $\mathrm{pH}$ (z-potential $=-28 \pm 1 \mathrm{MV},-30 \mathrm{mV}$ for APT ON -PEG-AuNPs and $-33 \pm 1 \mathrm{mV}$ for APT IN PEG-AuNPs with a PdI equal to 0.3 ) (Table 1). This stability was improved by the presence of PEG coating (Table 2) [2].

The APT AuNPs (APT ON PEG-AuNPs; APT IN PEG-AuNPs) were also characterized by TEM and have a spherical shape (Figure 1B) with a diameter of $10 \mathrm{~nm}$, for PEG-AuNPs confirmed by the hydrodynamic diameter measured by zetasizer (Table 2). The stability of APT AuNPs in solution, was monitored by the Localized Surface Plasmon (LSP) band at $523 \mathrm{~nm}$ (APT ON PEG-AuNPs) and $543 \mathrm{~nm}$ (APT IN PEGAuNPs). Analysis was carried out at pH 5.5 and electrolytic conditions. The synthesized APT AuNPs did shows an almost negligible change in the LSP band position over a period of three months (Figure S1). Some differences in term of LSP were observed between APT ON PEGAuNPs and APT IN PEG-AuNPs, due to different chemical behavior of APT AuNPs in function of size and shape respectively.

The successful immobilization of AQP2 aptamer onto and/or into PEG-AuNPs surface was also demonstrated by Raman spectroscopy (Figure $2 \mathrm{~A}-\mathrm{B}$ ). Moreover, we have access to some information regarding the aptamer structure with the Raman bands. Raman spectra of free gold nanoparticles (PEG-AuNPs) exhibit few bands assigned to the PEG molecules (The wide band observed around $1600 \mathrm{~cm}^{-1}$ on the Raman spectra is assigned to the water). The detection of the finger print of PEG-COOH at the AuNPs surface was demonstrated through the observation of the Raman bands at $1137 \mathrm{~cm}^{-1}, 1270 \mathrm{~cm}^{-1}$, and 1455 $\mathrm{cm}^{-1}$ due to the vibration of $\mathrm{C}-\mathrm{O}-\mathrm{H}, \mathrm{C}-\mathrm{O}-\mathrm{C}$ and $\mathrm{C}-\mathrm{O}$ chemical groups, respectively (Figure 2, black line). After the aptamer binding, many new bands can be observed for both grafting methods used (Figure 2 red line (APT ON PEG-AuNPs); Figure 2 green line (APT IN PEG-AuNPs) ). More details on the band assignment of APT are showed in the Table S1 [46-48]. Several bands are common to the grafted AuNPs but with different relative intensities: one peak at $838 \mathrm{~cm}^{-1}$ which corresponds to the vibration of the sugar, one at $1536 \mathrm{~cm}^{-1}$ which corresponds to the Adenine base and one at $1731 \mathrm{~cm}^{-1}$ which corresponds to ketones in the different bases of the aptamer [46]. Some bands are specific to the APT as the peak at $1058 \mathrm{~cm}^{-1}$ typical of phosphate group $\left(\mathrm{PO}_{4}^{3-}\right)$ [46] or the intense peak at $1371 \mathrm{~cm}^{-1}$ which corresponds to thymine base. Its high intensity is due to the good alignment of the aptamer with the $\mathrm{Au}$ surface of nanoparticles [49], in contrast to the lower signal recorded for the case of the free thymine DNA in water [50].

If we compare the Raman results of bioconjugation of APT with PEG-AuNPs (APT ON PEG-AuNPs; APT IN PEG-AuNPs) it confirms that the chemical and steric modification of the aptamer depending of the grafting method impacting the conformation of the aptamer. In particular the peak at $1528 \mathrm{~cm}^{-1}$ corresponding to Adenine and $\mathrm{NH}$ out plane, is more pronounced in APT IN PEG-AuNPs compared to APT ON PEG-AuNPs, confirming a different sterical disposition of
APT onto PEG-AuNPs. An effect SRS was observed in the range 300$300 \mathrm{~cm}^{1}$ correspponding to vibration $\mathrm{Au}-\mathrm{O}-\mathrm{C}, \mathrm{Au}-\mathrm{Cl}, \mathrm{O}-\mathrm{O}$ and 2500 $-3500 \mathrm{~cm}^{-1}$ due to $\mathrm{C}-\mathrm{C}$ aliphatic stretching (Figure 2 panel B). However, for each grafting method, we can assume that the aptamer different packing density and as a consequence its conformation will be largely influence by such parameters.

\section{Aptamer loading and release of APT ONPEG-AuNPs and APT IN PEG-AuNPs}

Aptamer technology was considered a novel promise in therapeutics for its targeting abilities [51]. However, its potential to improve drug loading and release from nano carriers has not been thoroughly analyzed. Recently Lee JB et al. have proposed a general approach for loading a large amount of thrombin proteins using DNA aptamer particles [52]; other authors, have developed a new strategy of drug-loading by designing drug specific aptamers to drive drugs into liposomes [53]. Of note, Dam et al. [54] showed thatloading of G-quadruplex DNA aptamer AS1411 onto AuNPs could be tuned by manipulating the charge of the ligands and the particle surface by $\mathrm{pH}$. They found that as the $\mathrm{pH}$ of the solution decreased, repulsion between negatively charged nucleic acids and the AuNPs surface decreased, which facilitated the covalent attachment of oligonucleotides on $\mathrm{Au}$ surface. The optimal $\mathrm{pH}$-tunable loading of a therapeutic G-quadruplex ligand under acidic medium, was applied on citrate-capped, colloidal AuNPs suggesting that this method can be general to a wide range of NPs. Based on these encouraging results, we have studied, for the first time, the synergic effect of a putative renal aptamer loading onto (method ON) and or /into (method IN) pegylated gold nanoparticles (PEG-AuNPs) under specific conditions and chemical conjugations.

\begin{tabular}{|c|c|}
\hline Sample & Endotoxin Units (EU/ml) \\
\hline PEG-AuNPs ( control) & ND \\
\hline APT ON PEG-AuNPs & $>1$ \\
\hline APT IN PEG-AuNPs & ND \\
\hline APT & $>1$ \\
\hline
\end{tabular}

Table 1: Endotoxin units detected by means of the LAL Chromogenic Endotoxin Quantitation kit (ND = not detectable; $>1=$ above detection limit of the assay).

\begin{tabular}{|c|c|c|c|}
\hline Synthetic product & $\begin{array}{c}\text { Zeta potential } \\
\mathbf{( m V )}\end{array}$ & $\begin{array}{c}\text { Hydrodynamic } \\
\text { diameter }(\mathbf{n m})\end{array}$ & Pdl \\
\hline PEG-AuNPs & $-28 \pm 1$ & $10 \pm 2$ & 0.3 \\
\hline APT ON PEG-AuNPs & $-30 \pm 1$ & $10 \pm 3$ & 0.3 \\
APT IN-PEG-AuNPs & $-33 \pm 1$ & $50 \pm 2$ & 0.3 \\
\hline
\end{tabular}

Table 2: z-potential and hydrodynamic diameter of PEG-AuNPs, APT on PEGAuNPs and APT IN PEG-AuNPs by carbodiimide and chelation chemistry.

\begin{tabular}{|c|l|}
\hline Raman peaks $\left(\mathrm{cm}^{-1}\right)$ & \multicolumn{2}{c|}{ Attribution } \\
\hline 833 & Désoxyribose \\
\hline 1058 & $\mathrm{PO}^{2-}$ \\
\hline 1137 & $\mathrm{COC}$ \\
\hline 1270 & $\mathrm{COH}$ \\
\hline 1371 & Thymine \\
\hline 1455 & $\mathrm{CO}$ \\
\hline 1536 & Adénine \\
\hline 1640 & $\mathrm{H}_{2} \mathrm{O}$ \\
\hline 1731 & $\mathrm{C}=0$ \\
\hline 1771 & $\mathrm{C}=0$ (carbodiimide bond) \\
\hline
\end{tabular}

Table S1: Raman band assignment for the APT and vibrational modes in the range spectral $800-1800 \mathrm{~cm}^{-1}$ 
In this study, after complexation of $\mathrm{AuCl}_{4}^{-}$with PEG diacid chains, APT was covalently bound onto complex after reduction of $\mathrm{NaBH}_{4}$ to obtain APT ON PEG-AuNPs. In this novel formulation APT assume a different chemical and sterical conformation compared to recent synthesis approach in which APT molecules reside inside the AuNP core ( APT IN PEG-AuNPs) [32,55]. The successful loading ratio of APT onto PEG-AuNPs, were evidenced by the characteristic absorption peaks at $260 \mathrm{~nm}$ from APT (Figure S2). The standard absorption of APT was plotted in the inset of figure (Figure S2) according to UVVis absorbance spectra of APT at various concentrations. The loading efficiencies were estimated to be $85 \%$ with $8.2 \mu \mathrm{g}$ present in $2.5 \times 10^{-8} \mathrm{~mol}$ of NPs (data not shown). A sustained APT release was observed in the first $5 \mathrm{~h}$ for both nanoparticles (APT ON PEG-AuNPs; APT IN PEG-
AuNPs) at $\mathrm{pH} 4$, which is highly favorable for delivery in the cancer cells [56]. We observed that the delivery of APT was favorite when the nano-formulation was obtained by method ON (APT ON PEGAuNps). Therefore, we supposed, that the release of APT was checked by a ionic equilibrium between $\mathrm{Au}$ (III)-complexes trapped into AuNPs by the hydrophobic interactions between PEG chaines and APT. As previously described, the mechanism by which acidic $\mathrm{pH}$ triggers drug release is probably associated to the presence of carboxylate groups in the chemical structure of PEG molecules [32]. Such groups become protonated at acidic $\mathrm{pH}$. This reduces the electrostatic interactions occurring between PEG and drugs that stabilize the APT ON PEGAuNPs structure (Figure 3).
A)

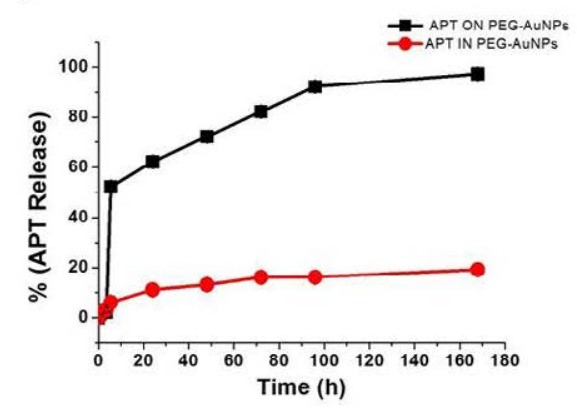

B)

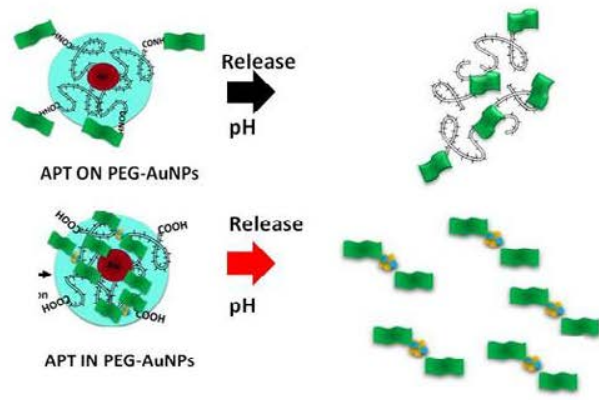

Figure 3: A) APT release percentage (\%) of APT ON PEG-AuNPs and APT IN PEG-AuNPs time in PBS $\left(37^{\circ} \mathrm{C}\right)$ at $\mathrm{pH}=4 \mathrm{~B})$ Schematic diagram of APT release under $\mathrm{pH}$ conditions.

A)

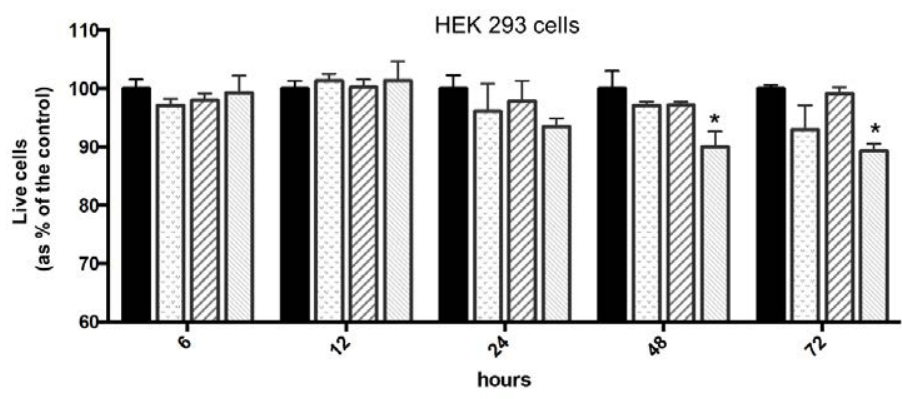

B)

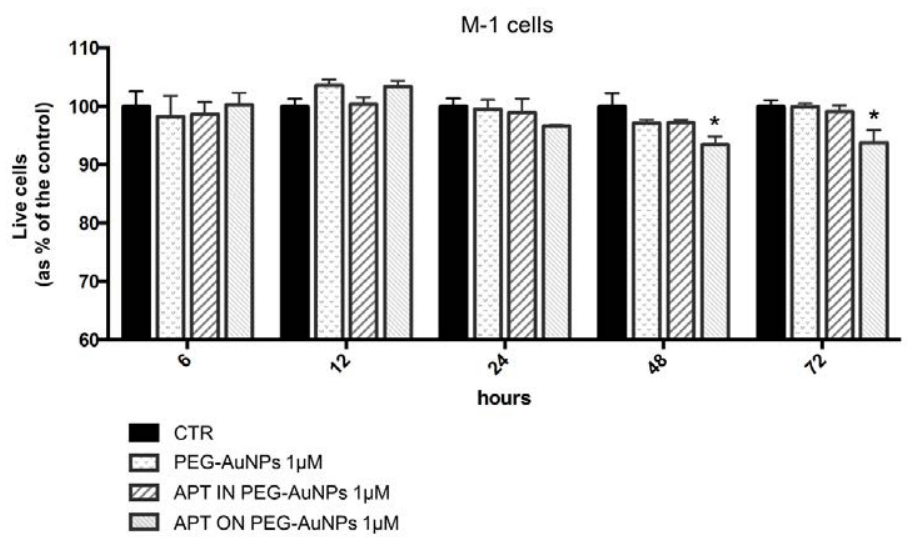

Figure 4: Cytotoxicity of APT ON PEG-AuNPs and APT IN PEG-AuNPs in renal cells: A) HEK 293 and B) M-1 cells were either left under basal condition (CTR) or exposed to PEG-AuNPs $(1 \mu \mathrm{M})$, APT ON PEG-AuNPs $(1 \mu \mathrm{M})$ and APT IN PEG-AuNPs $(1 \mu \mathrm{M})$ for $6,12,24,48$ and 72 hours at $37^{\circ} \mathrm{C}$ to evaluate NPs cytotoxicity using trypan blue exclusion assay. The cell viability was expressed as percentage of live cells vs total cells and normalized in respect to the CTR for each time point. Data were expressed as means \pm SEM of three independent experiments. $p<0.05$ was considered statistically significant. 
Citation: Arib C, Milano S, Gerbino A, Spadavecchia J (2018) Aptamer Grafting onto (on) and into (in) Pegylated Gold Nanoparticles: Physicochemical Characterization and In vitro Cytotoxicity Investigation in Renal Cells. J Nanomed Nanotechnol 9: 520. doi: 10.4172/2157-7439.1000520

Page 8 of 10

We suppose that, the ionic strength and protonation of APT onto PEG chains improve the water solubility of the APT molecules, taking advantage of the drug release from PEG-AuNPs. Indeed, a remarkable moiety of APT electrostatically binding onto dissociated complex is released, through PEG chain and diffusionphenomena [32,39].

\section{Cytotoxicity of apt on PEG-AuNPs and apt in PEG-AuNPs in renal cells}

Finally, the potential cytotoxic effect of these NPs on two different renal cell lines was evaluated. Human embryonic kidney and (HEK293) and mouse collecting duct (M1) cells [30] were exposed to medium containing vehicle alone, nanoparticles (PEG-AuNPs i.e. control), and nanoparticles produced with the aptamer either within (APT IN PEG-AuNPs) or outside (APT ON PEG-AuNPs) the nanoparticles. Whenever the aptamer was present the final concentration was $1 \mu \mathrm{M}$, which was the higher concentration possible according to the initial concentration of the solution containing the NPs. Cell viability was monitored for up to 72 hours. We found significant cell death, both for HEK293 (Figure 4A)

M-1 (Figure 4B) cells, after $24 \mathrm{~h}$ of exposure to APT ON PEGAuNPs. No significant cell death was evident for the other experimental conditions tested. The apoptosis inductor Staurosporin $(5 \mu \mathrm{M}$ for $6 \mathrm{~h})$ has been used as positive control in each experiment (data not shown).

The evaluation of cells viability under exposure of exogenous NPs is extremely important considering that, after NPs administration, they may migrate to different body sites through the systemic circulation. Indeed, several studied demonstrated NPs distribution and bioaccumulation within several organs such as liver, spleen, kidneys, brain or heart [57-59].

A number of recent studies have shown the impact of NPs accumulation in the kidneys. Chen et al. [60] described damage to proximal tubular cells in mice exposed to copper NPs while Wang et al. [61] Observed signs of glomerulonephritis and pathological degeneration in renal proximal convoluted tubules after oral titanium dioxide administration. These evidence together with our in vitro results highlight the urge for a carful risk assessment regarding all bioaccumulation sites within the body.

Here, we showed the ability of APT ON PEG-AuNPs to exert a significant albeit modest cytotoxic effect. However, further in vitro studies are needed to specifically investigate the mechanisms through which APT ON PEG-AuNPs induce cell death after $24 \mathrm{~h}$ exposure. The dissection of the cellular mechanisms leading to nanoparticlesinduced cell death might help the design of nano-molecules and nanocomplexes whose physicochemical characteristic shape, size, chemical or physical interactions do not interfere with cellular health. This will pave the way for a safer use of these new hybrid nanoparticles in biomedical approaches such as drugs delivery and gene therapy. Under this scenario, nanoparticles whose size allows renal filtration might represent a fascinating strategy to specifically target different parts of the nephron segment. In this study, we designed a putative aptamer against AQP2, which is a water channel responsible of facultative renal water reabsorption. Further investigations are further needed to exploit AQP2- nanoparticles based gene therapy to specifically rescue AQP2 physiological functions in a genetic disease whose main hallmark is impaired water reabsorption [62-66].

\section{Conclusion}

The success of this study was to provide the design, the synthesis and the characterization of new hybrid nanoparticles based on the incorporation of AQP2 aptamer (APT) PEGylated in gold nanoparticles under two chemical methodologies. Chemical physics characterizations were extensively achieved and fully elucidated the formation mechanism of the nanostructure and the APT conformational changes associated with such processes. Aptamer-conjugated PEG-AuNPs were assessed for in vitro study on two different renal cell lines, human embryonic kidney (HEK293) and mouse collecting duct cells (M-1), respectively. Based on different shape and size of different aptamer based gold nanoparticles, we will provide in the further, a selective study on several renal cells and therapeutic application thought phototermal (PTT) studies.

\section{Reference}

1. Khan I, Saeed K, Khan I (2017) Nanoparticles: Properties, applications and toxicities. Arab J Chem.

2. Spadavecchia J, Movia D, Moore C, Maguire CM, Moustaoui H, et al. (2016) Targeted polyethylene glycol gold nanoparticles for the treatment of pancreatic cancer: from synthesis to proof-of-concept in vitro studies. Int J Nanomed 11: 791-822.

3. Ahmad B, Hafeez N, Bashir S, Rauf A, Ur-Rehman M (2017) Phytofabricated gold nanoparticles and their biomedical applications. Biomedicine \& Pharmacotherapy 89: 414-425

4. Zhang RC, Sun D, Zhang R, Lin WF, Macias-Montero M, et al. (2017) Gold nanoparticle-polymer nanocomposites synthesized by room temperature atmospheric pressure plasma and their potential for fuel cell electrocatalytic application. Scien Rep 7: 46682

5. Spadavecchia J, Perumal R, Barras A, Lyskawa J, Woisel P, et al. (2014 Amplified plasmonic detection of DNA hybridization using doxorubicin-capped gold particles. Analyst 139: 157-164

6. Zhang S, Pelligra Cl, Feng X, Osuji CO (2018) Directed Assembly of Hybrid Nanomaterials and Nanocomposites. Adv Mat 30: 1705794.

7. Politi J, De Stefano L, Giardina P, Casale S, Rea I, et al. (2018) Hybrid Hydrophobin/Gold Nanoparticles: Synthesis and Characterization of New Synthetic Probes for Biological Applications. Springer 169-76.

8. Raj S, Jose S, Sumod US, Sabitha M (2012) Nanotechnology in cosmetics: Opportunities and challenges. J Pharm Bioallied Sci 4: 186-193.

9. Sinha SS, Paul DK, Kanchanapally R, Pramanik A, Chavva SR, et al. (2015) Long-range two-photon scattering spectroscopy ruler for screening prostate cancer cells. Chem Sci 6: 2411-2418.

10. Zuckerman JE, Davis ME (2013) Targeting therapeutics to the glomerulus with nanoparticles. Adv Chronic Kidney Dis 20: 500-507.

11. Yang RS, Chang LW, Wu JP, Tsai MH, Wang HJ, et al. (2007) Persistent tissue kinetics and redistribution of nanoparticles, quantum dot 705 , in mice: ICP-MS quantitative assessment. Environ Health Perspect 115: 1339-1343.

12. Choi HS, Liu W, Misra P, Tanaka E, Zimmer JP, et al. (2007) Renal clearance of quantum dots. Nat Biotechnol 25: 1165-1170.

13. Warheit DB, Webb TR, Reed KL, Frerichs S, Sayes CM (2007) Pulmonary toxicity study in rats with three forms of ultrafine-TiO2 particles: differential responses related to surface properties. Toxicology 230: 90-104.

14. Choi $\mathrm{CH}$, Zuckerman JE, Webster P, Davis ME (2011) Targeting kidney mesangium by nanoparticles of defined size. Proc Natl Acad Sci USA 108 6656-6661.

15. Song KM, Lee S, Ban C (2012) Aptamers and Their Biological Applications Sensors 12: 612-631.

16. Lee S, Pérez-Luna VH (2005) Dextran-Gold Nanoparticle Hybrid Material for Biomolecule Immobilization and Detection. Anal Chem 77: 7204-7211.

17. Priyadarshini E, Pradhan N (2017) Gold nanoparticles as efficient sensors in colorimetric detection of toxic metal ions: A review. Sens Actu B: Chem 238 888-902.

18. Medley CD, Bamrungsap S, Tan W, Smith JE (2011) Aptamer-Conjugated Nanoparticles for Cancer Cell Detection. Anal Chem 83: 727-734.

19. Oh SY, Heo NS, Shukla S, Cho HJ, Vilian ATE, et al. (2017) Development of 
Citation: Arib C, Milano S, Gerbino A, Spadavecchia J (2018) Aptamer Grafting onto (on) and into (in) Pegylated Gold Nanoparticles: Physicochemical Characterization and In vitro Cytotoxicity Investigation in Renal Cells. J Nanomed Nanotechnol 9: 520. doi: 10.4172/2157-7439.1000520

Page 9 of 10

gold nanoparticle-aptamer-based LSPR sensing chips for the rapid detection of Salmonella typhimurium in pork meat. Sci Rep 7: 10130

20. Shevchenko Y, Francis TJ, Blair DAD, Walsh R, DeRosa MC, et al. (2011) In Situ Biosensing with a Surface Plasmon Resonance Fiber Grating Aptasensor. Anal Chem 83: 7027-7034.

21. Bhardwaj V, Kaushik A (2017) Biomedical Applications of Nanotechnology and Nanomaterials. Micromach 8: 298

22. Peng L, Li BL, Zhou CW, Li NB, Setyawati MI, et al. (2018) Naked-eye" recognition: Emerging gold nano-family for visual sensing. Appl Mat Today 11: 166-188.

23. Gwinn MR, Vallyathan V (2006) Nanoparticles: Health Effects-Pros and Cons. Environ Health Perspect 114: 1818-1825.

24. Giri K, Shameer K, Zimmermann MT, Saha S, Chakraborty PK, et al. (2014) Understanding Protein-Nanoparticle Interaction: A New Gateway to Disease Therapeutics. Bioconjug Chem 25: 1078-90

25. Madeira A, Moura TF, Soveral G (2015) Aquaglyceroporins: implications in adipose biology and obesity. Cell Mol Life Sci 72: 759-71.

26. Kwon TH, Frøkiær J, Nielsen S (2013) Regulation of aquaporin-2 in the kidney: A molecular mechanism of body-water homeostasis. Kidney Res Clin Prac 32: 96-102.

27. Martins AP, Marrone A, Ciancetta A, Galán Cobo A, Echevarría M, et al (2012) Targeting aquaporin function: potent inhibition of aquaglyceroporin-3 by a gold-based compound. PLoSONE 7: e37435.

28. Jacques A, Lebrun C, Casini A, Kieffer I, Proux O, et al. (2015) Reactivity of Cys4 Zinc Finger Domains with Gold(III) Complexes: Insights into the Formation of "Gold Fingers". Inorg Chem 54: 4104-4113.

29. Jazayeri $\mathrm{MH}$, Amani $\mathrm{H}$, Pourfatollah $\mathrm{AA}$, Pazoki-Toroudi $\mathrm{H}$, Sedighimoghaddam B (2016) Various methods of gold nanoparticles (GNPs) conjugation to antibodies. Sensing Bio-Sensing Res 9: 17-22.

30. Stoos BA, Naray-Fejes-Toth A, Carretero OA, Ito S, Fejes-Toth G (1991) Characterization of a mouse cortical collecting duct cell line. Kidney Int 39 : 1168-1175.

31. Liu Q, Sacco P, Marsich E, Furlani F, Arib C, et al. (2018) Lactose-modified chitosan gold (III)-PEGylated complex -bioconjugates: from synthesis to interaction with targeted Galectin-1 protein. Bioconjug Chem.

32. Moustaoui H, Movia D, Dupont N, Bouchemal N, Casale S, et al. (2016) Tunable Design of Gold(III)-Doxorubicin Complex-PEGylated Nanocarrier. The Golden Doxorubicin for Oncological Applications. ACS Appl Mater Interfaces 8: 19946-19957.

33. Monteil M, Moustaoui H, Picardi G, Aouidat F, Djaker N, et al. (2017) Polyphosphonate ligands: from synthesis to design of Hybrid PEGylated Nanoparticles toward phototherapy studies. J Colloid Interface Sci $513: 205-213$

34. Morel AL, Giraud S, Bialecki A, Moustaoui H, de La Chapelle ML, et al. (2017) Green extraction of endemic plants to synthesize gold nanoparticles for theranostic applications. Frontiers Lab Med.

35. Marguerit G, Moustaoui H, Haddada MB, Djaker N, Chapelle ML, et al. (2018) Taxanes Hybrid Nanovectors: From Design to Physico-Chemical Evaluation of Docetaxel and Paclitaxel Gold (III)-PEGylated Complex Nanocarriers. Part Part Sys Charac 35: 1700299

36. Liu H, Jiang P, Li Z, Li X, Djaker N, et al. (2018) HIV-1 Tat Peptide-Gemcitabine Gold (III)-PEGylated Complex-Nanoflowers: A Sleek Thermosensitive Hybrid Nanocarrier as Prospective Anticancer. Parti Par Sys Charact 35: 1800082.

37. Javier DJ, Nitin N, Levy M, Ellington A, Richards-Kortum R (2008) AptamerTargeted Gold Nanoparticles As Molecular-Specific Contrast Agents for Reflectance Imaging. Bioconjug Chem 19: 1309-1312.

38. Capek I (2017) Polymer decorated gold nanoparticles in nanomedicine conjugates. Adv Colloid Interface Sci 249: 386-399.

39. Kammer MN, Olmsted IR, Kussrow AK, Morris MJ, Jackson GW, et al. (2014) Characterizing aptamer small molecule interactions with backscattering interferometry. Analyst 139: 5879-8584.

40. Hsu CL, Lien CW, Wang CW, Harroun SG, Huang CC, et al. (2016) Immobilization of aptamer-modified gold nanoparticles on $\mathrm{BiOCl}$ nanosheets: Tunable peroxidase-like activity by protein recognition. Biosens Bioelectron 75: 181-187.
41. Wang W, Ding X, He M, Wang J, Lou X (2014) Kinetic Adsorption Profile and Conformation Evolution at the DNA-Gold Nanoparticle Interface Probed by Dynamic Light Scattering. Anal Chem 86: 10186-10192.

42. Gomes de Castro MA, Höbartner C, Opazo F (2017) Aptamers provide superior stainings of cellular receptors studied under super-resolution microscopy. PLoSONE 12: e0173050.

43. Spadavecchia J, Casale S, Landoulsi J, Pradier CM (2014) Tuning the shape and size of hybrid gold nanoparticles by porphyrins using seed-mediated synthesis. Chem Phy Lett 609: 134-141.

44. Politi J, De Stefano L, Longobardi S, Giardina P, Rea I, et al. (2015) The amphiphilic hydrophobin $\mathrm{Vmh} 2$ plays a key role in one step synthesis of hybrid protein-gold nanoparticles. Colloids Surf B Biointerfaces 136: 214-221.

45. Pagba CV, Lane SM, Cho H, Wachsmann-Hogiu S (2010) Direct detection of aptamer-thrombin binding via surface-enhanced Raman spectroscopy. J Biomed Opt 15: 047006.

46. Pagba CV, Lane SM, Wachsmann-Hogiu S (2011) Conformational changes in quadruplex oligonucleotide structures probed by Raman spectroscopy. Biomed Opt Express 2: 207-217.

47. Sun Z, Wang Y, Wei Y, Liu R, Zhu H, et al. (2011) Ag cluster-aptamer hybrid: specifically marking the nucleus of live cells. Chem Commun 47: 11960-11962.

48. He H, Dai J, Duan Z, Zheng B, Meng Y, et al. (2016) Unusual sequence lengthdependent gold nanoparticles aggregation of the ssDNA sticky end and its application for enzyme-free and signal amplified colorimetric DNA detection. Sci Rep 6: 30878.

49. Schreier WJ, Schrader TE, Koller FO, Gilch P, Crespo-Hernández CE, et al. (2007) Thymine Dimerization in DNA is an Ultrafast Photoreaction. Science 315: 625-629.

50. Catuogno S, Esposito CL, de Franciscis V (2016) Aptamer-Mediated Targeted Delivery of Therapeutics: An Update. Pharmaceuticals 9: e69.

51. Kim J, Kim D, Lee JB (2017) DNA aptamer-based carrier for loading proteins and enhancing the enzymatic activity. RSC Advances 7: 1643-1645.

52. Plourde K, Derbali RM, Desrosiers A, Dubath C, Vallee-Belisle A, et al. (2017) Aptamer-based liposomes improve specific drug loading and release. J Control Release 251: 82-91.

53. Dam DHM, Lee H, Lee RC, Kim KH, Kelleher NL, et al. (2015) Tunable Loading of Oligonucleotides with Secondary Structure on Gold Nanoparticles through a pH-Driven Method. Bioconjugate Chem 26: 279-285.

54. Marguerit G, Ben Haddada M, Djaker N, de la Chapelle ML, Spadavecchia $J$, et al. (2017) Taxanes Hybrid Nanovectors: From design to physicochemical evaluation of docetaxel and paclitaxel gold (III)-PEGylated complex -nanocarriers. Par Par Sys Character.

55. Senapati S, Mahanta AK, Kumar S, Maiti P (2018) Controlled drug delivery vehicles for cancer treatment and their performance. Signal Transduct Target Ther 3: 7

56. Nemmar A, Hoet PH, Vanquickenborne B, Dinsdale D, Thomeer M, et al (2002) Passage of inhaled particles into the blood circulation in humans. Circulation 105: 411-414.

57. Hillyer JF, Albrecht RM (2001) Gastrointestinal persorption and tissue distribution of differently sized colloidal gold nanoparticles. J Pharm Sci 90: 1927-1936.

58. Oberdorster G, Sharp Z, Atudorei V, Elder A, Gelein R, et al. (2002) Extrapulmonary translocation of ultrafine carbon particles following wholebody inhalation exposure of rats. J Toxicol Environ Health A 65: 1531-1543.

59. Chen Z, Meng H, Xing G, Chen C, Zhao Y, et al. (2006) Acute toxicological effects of copper nanoparticles in vivo. Toxicol Lett 163: 109-120.

60. Wang J, Zhou G, Chen C, Yu H, Wang T, et al. (2007) Acute toxicity and biodistribution of different sized titanium dioxide particles in mice after oral administration. Toxicol Lett 168: 176-185.

61. Milano S, Carmosino M, Gerbino A, Svelto M, Procino G (2017) Hereditary Nephrogenic Diabetes Insipidus: Pathophysiology and Possible Treatment An Update. Int J Mol Sci 18.

62. Milano S, Gerbino A, Schena G, Carmosino M, Svelto M, et al. (2018) Human $\beta_{3}$-Adrenoreceptor is Resistant to Agonist-Induced Desensitization in Renal Epithelial Cells. Cell Physiol Biochem 48: 847-862. 
Citation: Arib C, Milano S, Gerbino A, Spadavecchia J (2018) Aptamer Grafting onto (on) and into (in) Pegylated Gold Nanoparticles: Physicochemical Characterization and In vitro Cytotoxicity Investigation in Renal Cells. J Nanomed Nanotechnol 9: 520. doi: 10.4172/2157-7439.1000520

Page 10 of 10

63. Procino G, Carmosino M, Milano S, Dal Monte M, Schena G, et al. (2016) $\beta(3)$ adrenergic receptor in the kidney may be a new player in sympathetic regulation of renal function. Kidney Int 90: 555-567.

64. Procino G, Gerbino A, Milano S, Nicoletti MC, Mastrofrancesco L, et al. (2015) Rosiglitazone promotes AQP2 plasma membrane expression in renal cells via a Ca-dependent/cAMP-independent mechanism. Cell Physiol Biochem 35: 1070-1085.
65. Procino G, Milano S, Carmosino M, Barbieri C, Nicoletti MC, et al. (2014) Combination of secretin and fluvastatin ameliorates the polyuria associated with X-linked nephrogenic diabetes insipidus in mice. Kidney Int 86: 127-138.

66. Procino G, Barbieri C, Carmosino M, Tamma G, Milano S, et al. (2011) Fluvastatin modulates renal water reabsorption in vivo through increased AQP2 availability at the apical plasma membrane of collecting duct cells. Pflugers Arch 462: 753-766. 